Copyright $@ 2010$ Institute of Electrical and electronics Engineers, Inc.

All Rights reserved.

Personal use of this material, including one hard copy reproduction, is permitted.

Permission to reprint, republish and/or distribute this material in whole or in part for any other purposes must be obtained from the IEEE.

For information on obtaining permission, send an e-mail message to stds-igr@ieee.org.

By choosing to view this document, you agree to all provisions of the copyright laws protecting it.

Individual documents posted on this site may carry slightly different copyright restrictions.

For specific document information, check the copyright notice at the beginning of each document. 


\title{
Functional Characteristics and Proposed Deployment Infrastructure of an Industrial Decision Support System within a Sugar Mill Crystallisation Stage
}

\author{
R. Dodd ${ }^{1}$, A. Chiou ${ }^{1}, X . \mathrm{Yu}^{2}$ and R.Broadfoot ${ }^{3}$ \\ ${ }^{1}$ Central Queensland University, Rockhampton, Queensland, Australia \\ Email:r.dodd@cqu.edu.au,a.chiou@cqu.edu.au \\ ${ }^{2}$ RMIT University, Melbourne, Victoria, Australia \\ Email:x.yu@rmit.edu.au \\ ${ }^{3}$ Queensland University of Technology, Brisbane, Queensland, Australia \\ Email:r.broadfoot@qut.edu.au
}

\begin{abstract}
This paper describes a deployment proposal and functional characteristics of a knowledge based supervisory support system (KBSSS) to provide expert knowledge in the control and management of the pan stage within a sugar mill. This decision support system utilises fragmented and diverse sources of information and unifies these into a cohesive structure to solve an important industrial control problem. Given the functional system characteristics, a deployment proposal for the embedding of the KBSSS within existing sugar mill infrastructure is presented.

The primary topic of this paper will be a description of: (1) the deployment proposal, (2) system process flows and (3) operational levels of the KBSSS.
\end{abstract}

\section{Introduction}

Raw sugar production from cane is a nominally continuous operation, with 120-168 hours of processing per week, extending over 20-25 weeks of the harvest season. The crystallisation section, often loosely referred to as the pan stage, is the most complex part of the factory process where there are several batch wise and continuous crystallisation steps taking place concurrently [1].

In current Australian practice, two operators are normally employed on the pan stage. There is considerable process interaction between the pan stage and centrifugal stage although management of the centrifugals is undertaken by different operators. The overall strategic management of the pan stage is quite difficult because of the very large number of process streams of varying compositions and crystal growth rate characteristics which must be managed [2]. Often the pan stage is managed in a sub-optimal manner because an overview of operations encompassing cane receival section, juice processing stations, the pan stage and centrifugal station of the sugar mill is not available.

The pressures on the Australian sugar industry to reduce the costs of sugar manufacture and increase the consistency of producing sugar of high quality require a smarter strategy for operation. Previous research [3] acknowledges that no conventional software engineering methods exist to provide an overall solution to this industrial problem due to its complexity, the wide variety of information sources required to be managed, overall management objectives, lack of adequate sugar mill crystallisation stage industrial process models and requirements for advisory strategies and supporting advice [4] to validate recommendations. Such wide and varied requirements are not easily managed and no such software based system for their unification currently exists to provide a solution.

Currently, there is no such supervisory control system for pan stage operations neither in the Australian sugar industry nor, as best as known to the collaborators, in the world sugar industry with literature only referring to a rudimentary advisory system for pan stage operations developed over three decades ago [5].

This paper is organised as follows. Section 2 presents the pan stage and KBSSS overview. Section 3 discusses the proposal for KBSSS deployment with focus on the fundamental information sources, end users and the software application. Section 4 discusses the process flow and operational levels of the system with a focus on end-users and their system interaction. Section 5 details the research undertaken to-date in realizing the proposed deployment architecture. The 
paper concludes with a summary presented in Section 6 and acknowledgements presented in Section 7.

\section{Pan Stage and KBSSS Overview}

The pan stage is a complicated feed-forward and feed-back series of operations superimposed upon a series of batch and continuous processing operations. In order to forward predict future pan stage operating conditions, a sequence of process models to describe the overall process is necessary. A series of industrial process models and supporting technology $[6,7,8,9]$ collectively working together to describe the primary pan stage inputs and outputs are required along with actual models of the internal workings of the pan stage itself.

An overall encompassing view of the various section of the factory is currently not available and operators are not able to predict future pan stage loadings with any form of assistance from existing factory systems. Furthermore, prediction facilities are not available to determine the consequence of operators actions other than the actual forward estimates the operators intuitively carry out.

The system specifications for key KBSSS features require that the system recommendations and expert advice provide four core control strategies [4]. The four core control strategies for the primary system output are:

1. Pan duty management;

2. Pan control strategy;

3. Pan schedule management; and

4. Stock tank management.

Additionally, these core control strategies are supported by a further two secondary system outputs. These secondary outputs are critical in providing reasoning and justifications for the recommended system advices. The secondary outputs are:

5. Prediction of future pan stage operating conditions for offered system recommendations compared to current operations; and

6. Explanatory and justification capabilities.

The KBSSS works in tandem with the pan stage operator and current pan stage computer control systems to perform the previously mentioned objectives and system advice. Figure 1 provides a visual depiction of interactions between the current systems and the operator. Existing pan stage infrastructure consists of sugar mill control system that interfaces directly with, and controls the pan stage operations. A pan stage operator interacts directly with this system.

The KBSSS is part of a cooperative control strategy and works in conjunction with the sugar mill control system and pan stage operators. It takes operator input along with information from the existing sugar mill control system via a real time parasitic data feed from the sugar mill control system databases. The KBSSS influences pans stage processes through the actions performed by the operators. The operators use advice and recommendations, in line with the reasoning process from the KBSSS, to influence pan stage operations through their interaction with the sugar mill control system.

\section{KBSSS Deployment Proposal}

The proposed implementation and deployment of the KBSSS is a cooperative pan stage solution working in conjunction with existing infrastructure and pan stage operators. The KBSSS application cannot exist as standalone software. The KBSSS requires information sources ranging from pan stage operators, knowledge base, fuzzy rule base and industrial process models of the pan stage to data from cane receival sections, juice processing station, the pan stage and centrifugal station sections of the sugar mill. Whilst existing sugar mill control system data is available in existing infrastructure, there is no readily available mechanism for facilitating the embedding of a secondary system such as the KBSSS.

Due to the data interaction that occurs as part of the KBSSS framework, the underlying rationale for proposed system deployment and integration within existing infrastructure has already been committed to indirectly, although not formally specified, as part of the system framework in previous research $[4,10,11]$.

This proposed deployment infrastructure is not a separate system to the KBSSS architecture and works in as part of the proposed system framework. The proposed deployment infrastructure can be decomposed into the following six levels, with each of these levels discussed in the following sub-sections:
1. Data sources;
2. Knowledge base;

EXISTING INFRASTRUCTURE

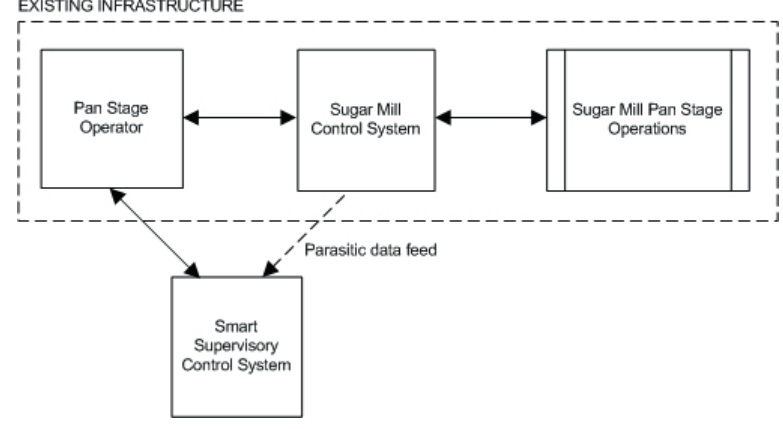

Figure 1. Smart supervisory control system interaction diagram. 
3. Industrial process models;

4. KBSSS software application;

5. System access; and

6. $\quad$ End users.

\subsection{Data Sources, Knowledge Base and Industrial Process Models}

The data source level consists of all information sources supplying data to the KBSSS. Local operating knowledge is elicited from end users though the consultation process and is archived for access in database format.

Sugar mill information from the cane receival, juice processing, pan stage and centrifugal sections of the factory is supplied to the data source level via parasitic data feeds from existing factory control systems infrastructure. There is no readily available solution to ensure correct information exchange between these two major systems. In order to provide a partial solution to this problem all databases in the KBSSS have been constructed with an industry standard relational database management system running on server technology. The relational database management system is separate from the KBSSS client application software. These databases exist on a database server allowing dedicated computer hardware performance in the sharing of KBSSS information sources.

Industrial process models are a major feature of the knowledge base. The underlying process model algorithms are hardcoded into the overall system yet flexibility is maintained by the ease of adaptability and customisation of input and output parameters through fuzzy logic localisation to account for real world operating conditions.

The underlying KBSSS knowledge base, fuzzy IfThen rule base and explanation knowledge base consists of knowledge from experts with specialised knowledge in the area of pan stage management and best practices. They provide the practical knowledge that is captured and modelled in the KBSSS. This knowledge is stored at the KBSSS knowledge base level.

\subsection{KBSSS Software Application}

A prototype system forms the KBSSS software application. The design, architecture and major supporting features are supported in previous research $[4,10,11]$. The KBSSS software application interacts directly with:
1. Pan stage experts providing information at the knowledge base level;

2. Pan stage operators providing information on real world conditions that are not part of the static predefined knowledge base. This user type is also providing with advice and recommendations on pan stage management and best practices by the KBSSS; and

3. Information sources from segments of the sugar factory through parasitic data feed from existing control system infrastructure.

The final system output is presented to pan stage operators to aid in the pan stage decision making process.

\subsection{System Access and End Users}

The access level specifies how end users can access the KBSSS. The system is developed for use by pan stage operators and support staff within the sugar mill pan stage environment. For practicality, the software would run on hardware systems located in the pan stage control centre, due to the KBSSS functioning in a human cooperative support manner in the decision making process. The KBSSS contains no automated feedback loop for pan stage control. Interaction with the pan stage is through the actions taken by pan stage operators in accordance with the KBSSS advice and recommendations. Pan stage operators have computer based access to control systems as part of their existing duties. Factory automation uses computer based control with computer infrastructure and computer networking facilities already existing as part of standard factory operations.

End users require database access permissions to KBSSS system databases. Modification of these critical database system systems without the use of KBSSS software application is not permissible. Access permissions to factory control system data, used by the KBSSS through the parasitic data feed, are also required. Additionally, such access is only to be permitted in a read-only fashion to prevent data modification of critical factory control system information.

\section{Process Flow and Operation Levels}

The KBSSS is supported by the seven key databases presented in Figure 2. During KBSSS operation, the access and modification of data within these information sources occurs at two levels of operation. These levels are: 


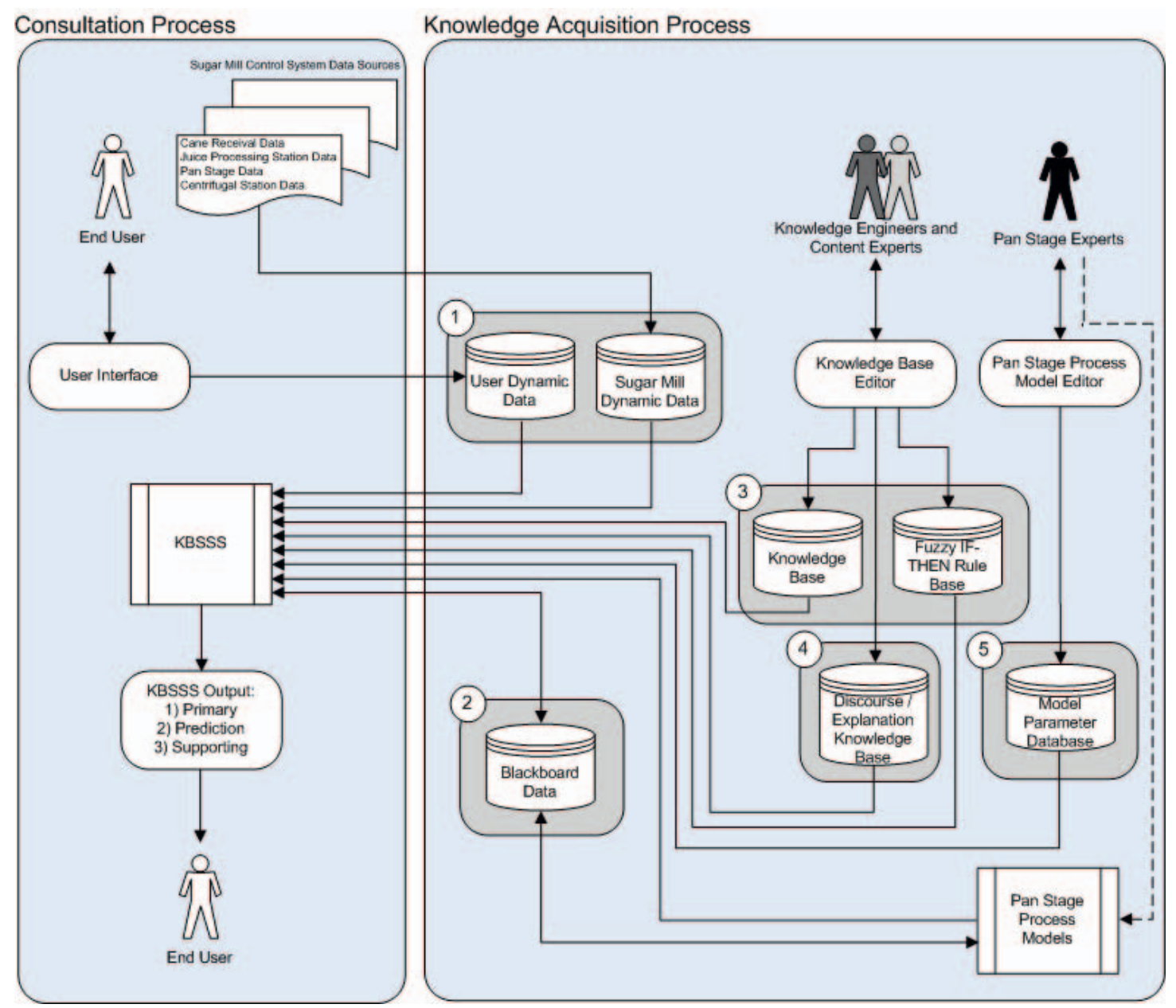

\section{Figure 2. KBSSS process flows showing interaction of the user groups with the information sources for the knowledge acquisition and consultation processes.}

1. Knowledge acquisition process, and

2. Consultation process.

Through the knowledge acquisition process knowledge engineers, content experts and pan stage experts model pan stage knowledge. The information extracted from this process is stored as part of the KBSSS data sources. The consultation process level allows end users to interact with the KBSSS for advice, recommendations and supporting information in the provision of best practices and management for pan stage operations.

Systems users fall into four distinct categories. End users interact in a consulting fashion with the KBSSS in the provision of its advice. Knowledge engineers and content experts develop and maintain the system knowledge and discourse bases used in modelling expert knowledge and explanatory capabilities. Pan stage experts develop and maintain the dynamic industrial pan stage process models and their default model parameters used in the prediction of future operating conditions. The final system group is a pseudo-user interaction maintained by the sugar mill control system data sources. Information sources from cane receival, juice processing station, pan stage and centrifugal station are real world information sources that the KBSSS draws from during its operation.

The seven database systems used in the KBSSS are grouped depending upon functionality. This clustering is:

1. Real world databases consisting of user dynamic data and sugar mill dynamic data;

2. System supporting database consisting of blackboard database;

3. Knowledge bases consisting of the KBSSS knowledge base and fuzzy If-Then rule base;

4. Discourse base comprising the discourse/explanation knowledge base; and

5. Model parameter database comprised of databases storing default pan stage process model parameters.

These functional groups are annotated with the same clustering levels in Figure 2. This figure 
illustrates interaction with the KBSSS user groups as part of the consultation and knowledge acquisition phases of operation.

\subsection{Knowledge Acquisition Level}

The knowledge acquisition process is outlined in Figure 2. The users at this level are the content experts, knowledge engineers and pan stage experts.

The knowledge bases are developed by the knowledge engineers to establish relationships in pan stage best practices and management. Knowledge is translated into fuzzy membership functions as part of the process of building fuzzy If-Then rules to model pan stage relationships. This transformation occurs through the use of the knowledge base editor. Content engineers then establish the discourse relationships between the fuzzy rules and explanations.

The industrial pan stage process models $[6,7,8,9]$ are initially developed by pan stage experts and integrated within the KBSSS software application. This relationship is indicated by the broken line in Figure 2 since the implemented pan stage process models are not able to be directly modified and exist as software. These models form part of system knowledge base detailing the pan stage process and dynamic interrelations between segments in the complex factory environment. The pan stage experts, as domain experts, also develop the industrial process model default parameters that are used as part of the pan stage process models. These parameters are transformed through the pan stage process model editor and saved to the appropriate model databases. These defaults are overridden by the fuzzy If-Then rules to provide adaption to account for real world operating conditions and local trends.

Information sources from the real world databases are fundamentally different in comparison to the static knowledge bases. Whereas the knowledge bases are essentially static over the lifetime of the KBSSS, given allowance for maintenance procedures, the real world databases provide information sources that are dynamic and vary with time. Dynamic user data is elicited in consultation with the end user in providing system advice and recommendations. Information provided by end users has a much greater lifespan than real time control system data which is essentially a constant information stream. Information sources from the sugar mill control system provides real time information on cane receival, juice processing, pan stage and centrifugal sections of the sugar mill through a parasitic data link to existing sugar mill infrastructure. Given the changing nature of these information sources, updated information from these sources is required each time the KBSSS carries out its processing regime in the generation of advice, recommendations, supporting explanations and the future forecast of pan stage operating conditions.

An additional key information source is the system supporting database composed of the blackboard system. This shared working memory stores partial solutions in building the forward forecast of pan stage operations with information flows controlled by the blackboard scheduling system.

\subsection{Consultation Process Level}

The consultation process as shown in Figure 2 involves interaction between the end user, sugar mill control system data sources and the KBSSS. The end user supplies real world information on current operating conditions and trends. This information, along with data provided by the sugar mill control system, is captured and stored for use in both the inference process and by the industrial pan stage process models.

User supplied information from the consultation process, along with pan stage control system data is used in the inference process and prediction of future pan stage operating conditions by the industrial pan stage process models. The results of this process are the primary systems recommendations with supporting outputs [4]. The consultation process ends with the generation of the final system outputs. Based upon the system recommendations and reasoning presented, the end user will determine which operational decisions should be made using the pan stage forecast of future operating conditions as supporting information in the decision making process.

\section{Proposed Deployment Realisation}

In the implementation of the proposed deployment structure, the following items from Figure 2 have been prototyped as proof of concept, with a research partnership involving Racecourse sugar mill located in Mackay, Queensland, Australia.

The design of the system framework and software architecture for the KBSSS application has been undertaken and is supported in previous research [4, $10,11]$.

Industrial pan stage process models $[6,7,9]$ and supporting technologies [8] have been developed, accompanied by model parameter databases and editing facilities. Data from cane receival, juice processing, pan stage and centrifugal sections of the sugar mill have been interfaced using historic archived database information as a precursor to the proposed 
parasitic data feed of real time sugar mill control system data.

Control system data from the 2003 crushing season has been used in the development of a series of industrial process models collectively working together to describe the primary pan stage inputs and outputs along with actual models of the internal workings of the pan stage itself. A blackboard system, used as a shared working memory for these process models, has also been completed.

Partial but varying levels of completion have occurred for the other components/data sources listed in Figure 2. It is envisaged that full implementation and testing of the proposed deployment architecture will be undertaken as part of a follow-on research project.

\section{Summary}

This paper presents a proposal for a KBSSS deployment for use within pan stage operations of a sugar mill. The role and embedding of this system into existing infrastructure is presented with the major information sources, required for system operation, detailed. The major data flows, data sources and contributing components are also highlighted with reference to user interaction.

The KBSSS is an innovative architecture to unify fragmented systems of data from pan stage operators, fuzzy rule base, developed industrial process models of the pan stage and information sources across varying sections of the sugar mill to solve an important industrial control problem. This system seeks to provide a unifying structure to assist pan stage operators in the decisions making process for pan stage control and management.

\section{Acknowledgements}

The project contributors would like to thank the staff at Racecourse Sugar Mill, Mackay, Australia for their cooperation and support. This project was supported by an Australian Research Council linkage grant. The funding assistance provided by the Sugar Research Institute, Brisbane, Australia is also acknowledged and appreciated.

\section{References}

[1] R. Broadfoot and A. Beath, "Modelling of batch sugar centrifugal performance", in Proceedings of 26th Australasian Chemical Engineering Conference, 1988, pp.294-303.
[2] K. Miller and R. Broadfoot. "Crystal growth rates in high grade massecuite boilings", in Proceedings of the Australian Society of Sugar Cane Technologists Conference, 1997, pp.441-447.

[3] R. Dodd, "A knowledge based supervisory support system for pan stage operations in a sugar mill", Ph.D dissertation, Royal Melbourne Institute of Technology University, Melbourne, Victoria, Australia, 2009.

[4] R. Dodd, A. Chiou, X. Yu and R. Broadfoot. "Framework for a smart supervisory control system for a sugar mill crystallisation stage", in Proceedings of the 6th IEEE International Conference of Industrial Informatics, 2008, pp. 463-468.

[5] J. Frew and P. Wright, "Sugar crystallisation: a pan stage advisory scheme", in Proceedings of Queensland Society of Sugar Cane Technologists, 1976, pp. $191-198$.

[6] R. Dodd, R. Broadfoot, X. Yu and A. Chiou, "Empirical pan modelling of vacuum pans for a sugar mill crystallization stage", in Proceedings of Australian Society of Sugar Cane Technologists, 2005, pp.423-436.

[7] R. Dodd, R. Broadfoot, X. Yu and A. Chiou, "Process models for a sugar mill crystallisation stage knowledge based supervisory support system", in Proceedings of I*PROMS Virtual International Conference, 2008, pp. 1-6.

[8] R. Dodd, R. Broadfoot, A. Chiou, and X. Yu, "Dynamic allocation of predicted quantities to forecast intervals for pan stage supervisory support system process models", in Proceedings of the 2009 IEEE International Conference on Industrial Technology, 2009, pp. 1-6.

[9] R. Dodd, R. Broadfoot, A. Chiou, and X. Yu, "Pan stage steady state flow model for integration within a knowledge based supervisory support system", in Proceedings of the 2009 IEEE International Conference on Industrial Technology, 2009, pp. 1-6.

[10] R. Dodd, R. Broadfoot, X. Yu and A. Chiou, "'Development of smart supervisory control system in a sugar mill crystallisation stage", in Proceedings of I*PROMS Virtual International Conference, 2005, pp.527-534.

[11] X. Yu, A. Chiou and R. Dodd, "A novel decision support framework for industrial processes", in Proceedings of the 31st Annual Conference of IEEE Industrial Electronics Society, 2005, pp. 2210-2215. 